\title{
Distributed Load Frequency Control of Electrical Power Networks via Iterative Gradient Methods
}

\author{
Toru Namerikawa and Taichiro Kato
}

\begin{abstract}
This paper discusses a distributed control for load frequency of electrical power networks based on iterative gradient methods. The control objective is to minimize the quadratic cost function of load frequency control problem, and we apply the distributed control methodology by using iterative gradient methods to complicated large scale electrical power networks. Iterative gradient methods have good flexibility and they are effective for complicated and time-varying distributed power networks. Several numerical simulation results of distributed power network systems including distributed generations, batteries, and renewable energies in order to show the effectiveness of the load frequency control compared with conventional decentralized control and centralized control.
\end{abstract}

\section{INTRODUCTION}

Electrical power energy problems and global warming have become one of the hottest worldwide social problems. Therefore, a lot of distributed generations such as the photo voltaic and wind power generations, the biomass power generations, and the co-generations, are going to be installed in large scale power network systems from viewpoints of energy conservation and the cost reduction. At the same time, it is well-known that they have adverse affects on system frequency and fluctuation of voltage in power systems. Hence, it is necessary to explicitly control every generation cooperatively and optimally and ensure safety. In 70 's, the optimal control of the electric power system was deeply studied e.g., [1]. Recently, system frequency control in power networks installing photo voltaic and wind power generations, battery energy storage system, and heat pump system, has been studied by [2].

By the way, the decision making problems to use different information concerning underlying uncertainties has been studied since the 1950's. Some of typical problems in these fields are called game problem and team problem et.al. Relations with the distributed control and decision making was strengthened in the 1970's, and distributed control was studied actively, and some special types of team problems were solved by [3], [4] . Recently, the concern for the distributed control has risen because many new large scale systems appeared that can apply a distributed control theory. The problem area has become recently gain renewed interest and researched by [5]-[7].

In this paper, we propose the system frequency control method by using distributed control based on the iterative gradient methods to the distributed electric power network

T. Namerikawa and T. Kato are with Department of System Design Engineering, Keio University, 3-14-1 Hiyoshi, Kohoku-ku, Yokohama 223-8522 JAPAN namerikawa@sd.keio.ac.jp, taichiro@nl.sd.keio.ac.jp systems introducing the distributed generations. The varying state feedback gains are tuned and optimized repeatedly using iterative gradient methods. The advantage of this method is to change controller of new system and its adjacent system when the system configuration is changed. Finally, The proposed method is applied to the frequency control on the electric power networks with the distributed generations, and effectiveness is shown by the simulation.

The following notations are used in this paper. $\mathbb{Z}_{+}$is sets of nonnegative integers. $\mathbb{R}^{n}$ shows the real number space of the $n$ dimension. $\mathbf{E}$ is a expectation operator. $\operatorname{Tr} A$ shows the trace of $A$. $Q>0(Q \geq 0)$ shows that $Q$ is a (semi-)positive definite matrix.

\section{DistRibuted SySTEMS}

We consider electric power networks which consist of more than two subsystems. Assume that there exist $N(\geq 2)$ electric power systems and $i$ th subsystem $(i=1, \cdots, N)$ is given by the following equation

$$
x_{i}(t+1)=\sum_{j=1}^{N} A_{i j} x_{j}(t)+B_{i} u_{i}(t)+w_{i}(t)
$$

where $t \in \mathbb{Z}_{+}, x_{i}(t) \in \mathbb{R}^{n_{x i}}$ is a state of systems $i, A_{i i} \in$ $\mathbb{R}^{n_{x i} \times n_{x i}}$, if $j \neq i A_{i j} \in \mathbb{R}^{n_{x j} \times n_{x j}}, u_{i}(t) \in \mathbb{R}^{n_{u i}}$ is a control signal of system $i, w_{i}(t) \in \mathbb{R}^{n_{w i}}$ is a white noise with variance $\mathrm{W}$, and $w_{i}(t)$ is independent of $x_{i}(s)$ for $s \leq t$.

If $i$ th subsystem in the electric power system and $j$ th subsystem are connected, then $i$ and $j$ are neighbors and it is written as $(i, j) \in E$ If they are not connected, this topology can be expressed by

$$
A_{i j}=0 \quad \text { if }(i, j) \notin E
$$

$A_{i j}$ shows how electric power system $i$ has an influence on $j$. The matrix A of power networks whose structure is shown in Fig. 1 can be expressed as the following equation.

$$
A=\left[\begin{array}{cccc}
A_{11} & A_{12} & 0 & 0 \\
A_{21} & A_{22} & A_{23} & 0 \\
0 & A_{32} & A_{33} & A_{34} \\
0 & 0 & A_{43} & A_{44}
\end{array}\right]
$$

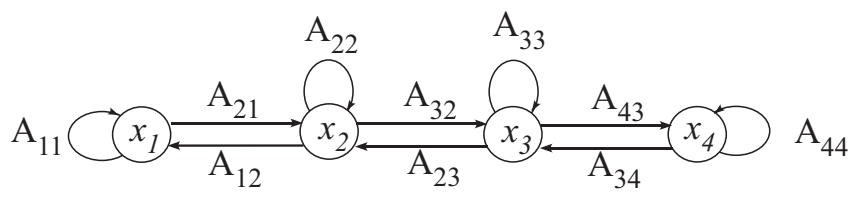

Fig. 1. An example of the distributed systems 
The electric power network that consists of $N$ subsystems can be shown as an equation (4).

$$
x(t+1)=A x(t)+B u(t)+w(t)
$$

where $t \in \mathbb{Z}_{+}, x(t) \in \mathbb{R}^{n_{x}}$ is state, $u(t) \in \mathbb{R}^{n_{u}}$ is control signal, $w(t) \in \mathbb{R}^{n_{w}}$ is white noise, and satisfy following equations.

$$
\begin{gathered}
\mathbf{E} w(t) w(s)^{T}=W \delta_{t s} \\
\mathbf{E} w(t) x^{T}(s)=0, \quad \text { if } t \geq s \\
\delta_{t s}=1 \quad \text { if } t=s, \quad \delta_{t s}=0 \quad t \neq s
\end{gathered}
$$

It is assumed we can obtain information of all elements of the state vectors. This system uses the following state feedback as a control input.

$$
u(t)=-L x(t)
$$

When we consider a distributed setup here, we limit feedback matrices to have a structure that matches the system. Therefore, only the measurement value in the adjoining electric power system is required to calculate control input $u_{i}(t)$ in electric power system $i$. It is assumed that the electric power system of $N$ systems has the following feedback matrix.

$$
L=\left[\begin{array}{cccc}
L_{11} & L_{12} & \ldots & L_{1 N} \\
L_{21} & L_{22} & \ldots & L_{2 N} \\
\vdots & \vdots & \ddots & \vdots \\
L_{N 1} & L_{N 2} & \ldots & L_{N N}
\end{array}\right], \quad L_{i j}=0 \text { if }(i, j) \notin E
$$

With this setup the dynamics closed-loop matrix $A-B L$ has the same structure as $A$.

\section{Distributed COnTROl Based on Iterative GRADIENT METHODS}

To decide feedback matrix $L$, the cost function is defined

$$
J(L)=\boldsymbol{E}\left(|x|_{Q}^{2}+|u|_{R}^{2}\right)
$$

We define $|x|_{Q}^{2}=x^{T} Q x$ and $|u|_{R}^{2}=u^{T} R u$, and assume $Q$ and $R>0$.

$$
L_{k+1}=L_{k}-\gamma \nabla_{L} J
$$

It is assumed that $\gamma$ is small enough. Feedback matrix $L$ can be calculated repeatedly by using gradient method. Next, the gradient of $J(L)$ was given by the following proposition 1 .

Proposition 1: [5]

Given matrices $A$ and $B$, consider $L$ such that $A-B L$ has eigenvalues inside the unit circle. It is assumed that stationary stochastic process satisfies (4)-(7) and (8). The gradient of $J(L)$ defined by (10) can be shown by the next expression.

$$
\nabla_{L} J=2\left[R L-B^{T} P(A-B L)\right] X
$$

where $X$ and $P$ satisfy Lyapunov equations, respectively.

$$
\begin{aligned}
& X=(A-B L) X(A-B L)^{T}+W \\
& P=(A-B L)^{T} P(A-B L)+Q+L^{T} R L
\end{aligned}
$$

What we want to know is not only the gradient of $\nabla_{L} J$ but the distributed method of calculation. To know distributed method, we introduce the adjoint system and the following was already obtained proposition 2 .

\section{Proposition 2: [5]}

Stationary stochastic process $\lambda$ is defined by back wards iteration under the condition of proposition 1 .

$$
\lambda(t-1)=(A-B L)^{T} \lambda(t)-\left(Q+L^{T} R L\right) x(t)
$$

where $x(t)$ are states of the original system. Then, the gradient of $J$ is expressed as the following equation.

$$
\nabla_{L} J=2\left(R L \boldsymbol{E} x x^{T}+B^{T} \boldsymbol{E} \lambda x^{T}\right)
$$

Proposition 2 gives a way of estimating an update direction for the feedback matrix $L$. But, we can't calculate in a distributed way. Because the covariance between all states and all adjoint states need to be determined under proposition 2. With appropriate prediction for the gradient, we can calculate feedback matrix $L$ in a distributed way. To do this we restrict $Q$ and $R$ to be block-diagonal, which fit the size of the state space and number of inputs of each agent, respectively.

The system $i$ needs to estimate $\lambda_{i}$ by using only local information of adjacent system. Hence, adjoint state of system $i$ is expressed as (17).

$$
\lambda_{i}(t-1)=\left[A_{L}^{T} \lambda(t)\right]_{i}-\left[\left(Q+L^{T} R L\right) x(t)\right]_{i}
$$

Each system is considered to be able to know both $A_{L i j}$ when system $i$ and system $j$ are adjacent by previous assumptions. The equation (17) can be expressed as in (18).

$$
\lambda_{i}(t-1)=\sum_{j \in E_{i}}(A-B L)_{j i}^{T} \lambda_{j}(t)-\left(Q_{i} x_{i}(t)-\sum_{j \in E_{i}} L_{j i}^{T} R_{j} u_{j}(t)\right)
$$

This means that the adjoint state equation (17) can be simulated in each agent using only local information. As $L$ satisfies (9), the actual update direction of the feedback matrix also need to satisfy this structure. Therefore, the gradient of $\nabla_{L} J$ is considered to be the subspace with the same structure. Letting $G$ be updated, the following equation is obtained.

$$
\begin{aligned}
& G_{i j}=\left(\nabla_{L} J\right)_{i j} \quad \text { if }(i, j) \in E \\
& G_{i j}=0 \quad \text { otherwise }
\end{aligned}
$$

Assuming the projected gradient $G$ is not $0,-G$ decent direction of $J(L)$. This means that system $i$ has only to determine the gradient in the blocks corresponding to the neighboring system. Therefore, it is necessary to estimate both $\left(R L \boldsymbol{E} x x^{T}\right)_{i j}$ and $\left(B^{T} \boldsymbol{E} \lambda x^{T}\right)_{i j}$ from information in the adjoining systems. The first term of the gradient given by proposition 2 can be simplified to the next expression.

$$
\left(R L \boldsymbol{E} x x^{T}\right)_{i j}=-R_{i} \boldsymbol{E} u_{i} x_{j}^{T}
$$

The second term can be rewritten as the next equation with the assumption of the structure on $B$.

$$
\left(B^{T} \boldsymbol{E} \lambda x^{T}\right)_{i j}=B_{i}^{T} \boldsymbol{E} \lambda_{i} x_{j}^{T}
$$

We can also estimate this term from the adjoining systems. With this analysis, it shows that we can estimate the update of 
a feedback matrix using only local information. The method is summarised in the following update scheme.

Algorithm 1: At time $t_{k}$, let the state feedback $u(t)=$ $L^{(k)} x(t)$. To update the feedback matrix in system $i$.

1) Simulate the state $x_{i}(t)$ of the (4) for $t=t_{k}, \ldots, t_{k}+N$ by exchanging states information with states of neighboring system.

$$
x_{i}(t+1)=\sum_{j \in E_{i}}(A-B L)_{i j} x_{j}(t)+w_{j}(t)
$$

2) Simulate adjoint state $\lambda_{i}(t)$ of the (17) for $t=t_{k}, \ldots, t_{k}+$ $N$ in back wards direction by exchanging states information with states of neighboring system.

$$
\lambda_{i}(t-1)=\sum_{j \in E_{i}}(A-B L)_{j i}^{T} \lambda_{j}(t)-\left(Q_{i} x_{i}(t)-\sum_{j \in E_{i}} L_{j i}^{T} R_{j} u_{j}(t)\right)
$$

3) $\boldsymbol{E} u_{i} x_{j}^{T}$ and $\boldsymbol{E} \lambda_{i} x_{j}^{T}$ are calculated by using all states of adjoining systems $j$.

$$
\begin{aligned}
& \left(\boldsymbol{E} u_{i} x_{j}^{T}\right)_{e s t}=\frac{1}{N+1} \sum_{t=t_{k}}^{t_{k}+N} u_{i}(t) x_{j}(t)^{T} \\
& \left(\boldsymbol{E} \lambda_{i} x_{j}^{T}\right)_{e s t}=\frac{1}{N+1} \sum_{t=t_{k}}^{t_{k}+N} \lambda_{i}(t) x_{j}(t)^{T}
\end{aligned}
$$

4)The estimate of $i, j$ block of the gradient becomes

$$
G_{i j}=-2\left[R_{i}\left(\boldsymbol{E} u_{i} x_{j}^{T}\right)_{e s t}+B_{i}^{T}\left(\boldsymbol{E} \lambda_{i} x_{j}^{T}\right)_{e s t}\right]
$$

5)When assuming some step $\gamma$, update of each neighboring system $j$ is expressed as $L_{i j}^{(k+1)}=L_{i j}^{(k)}-\gamma G_{i j}$

6) $t_{k+1}=t_{k}+1$, increase $k$ by one and go to 1 )

\section{SySTEM FREQUENCY CONTROL ON ELECTRIC POWER NETWORK}

\section{A. Electric power system model}

We consider electric power network shown in Fig.2. It is assumed that the composition of four electric power systems are same, there are gas turbine generators and wind power generations in the system, and the power supply is done to the electric power demand with these power generating machines. To bring frequency deviation $\Delta f$ of the system frequency close to 0 by using the TBC method as a frequency control in the electric power system in consideration of tieline flow. The gas turbine output is controllable [8].

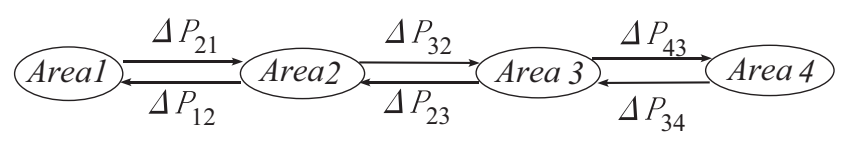

Fig. 2. Power network

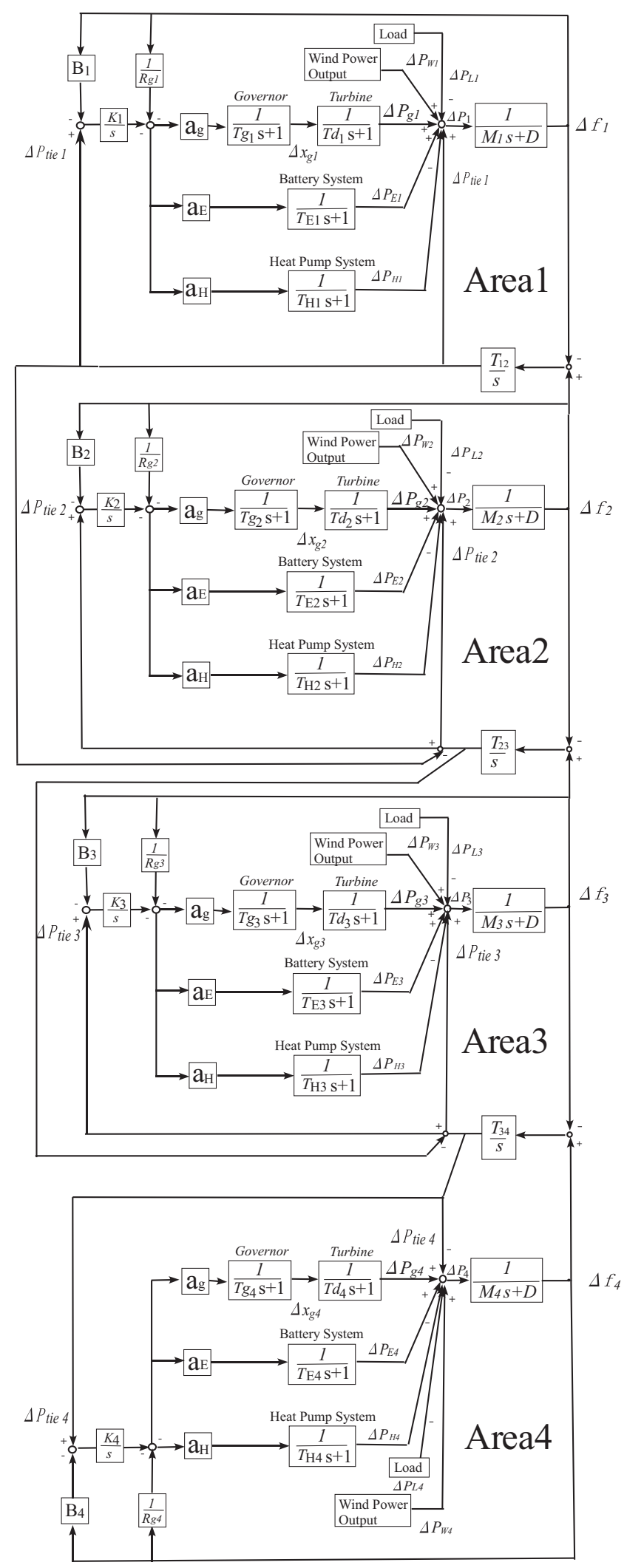

Fig. 3. Frequency analysis model of Power network

We apply the electric power network in Fig.2 to the frequency analysis model as in Fig.3. Each system capacity is assumed to be same $40[\mathrm{MW}]$. 
In this paper, we consider distributed mass loads, which are battery electric storage systems (electric vehicle etc.) and electric water heaters (heat pump etc.), are controllable. We assume battery electric storage systems around $5 \%$ of the system capacity and electric water heaters are around $15 \%$ respectively. We assume the heat pump group and battery electric storage systems as first-order systems, and don't think of the capacity of the heat pump group and battery electric storage system, and all system operate a constant characteristic. Here time constant $T_{H}$ is assumed to be 4 [s], and $T_{E}$ is assumed to be $0.2[\mathrm{~s}]$.

$\Delta P_{g i}$ is an output of gas turbine generator, $\Delta x_{g i}$ is a governor input of gas turbine generator, $\Delta P_{W i}$ is an output of wind power generation, $\Delta P_{L i}$ is a load fluctuation except controllable load, $\Delta P_{E i}$ is an output of battery electric storage system, $\Delta P_{H i}$ is an output of electric water heater, $\Delta P_{t i e_{i}}$ is an tie-line power flow deviation in Fig.3. $\Delta P_{i}$ of the (27) shows the power generation electric power of area $i$ and the supply error margin of power consumption. Frequency deviation $\Delta f_{i}$ can be calculated in the block shown from the supply error margin that occurs in the system in Fig.4. when it is assumed that all generators in area is completely synchronous driving, system can be expressed as one equivalent model like Fig.4.

$$
\Delta P_{i}=\Delta P_{g i}+\Delta P_{W i}-\Delta P_{L i}+\Delta P_{t i e} i+\Delta P_{E i}-\Delta P_{H i}
$$

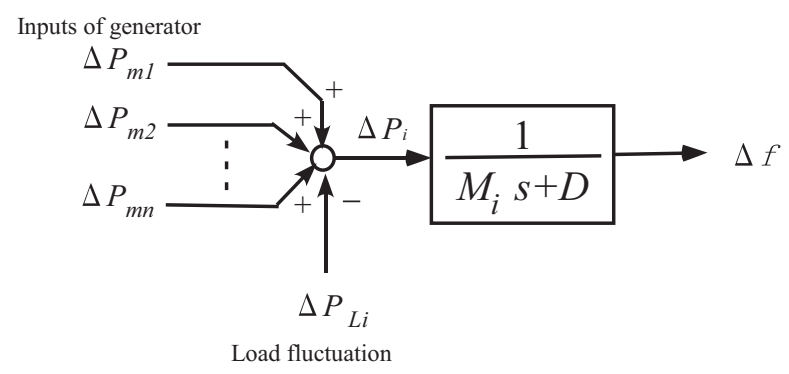

Fig. 4. Equivalent generator model of multi-generator system

As the tie-line power flow deviation of are $i$ is expressed $\Delta P_{t i e_{i}}=T_{i j}\left(\Delta f_{j}-f_{i}\right)$ when the adjoining area is $j$. AR which is a regional demand is written as $A R_{i}=\Delta P_{t i e_{i}}$ $B_{i} \Delta f_{i}$, and we defined as $U_{A R_{i}}=\int A R_{i} d t$. Moreover, the LFC signal is made by the PI control, and in the ratio of each generator. $a_{g}, a_{E}$, and $a_{H}$ satisfy the ratio of the system capacity of each gas turbine, battery electric storage system, and electric water heater. They also satisfy following assumption $a_{g}+a_{E}+a_{H}=1$. $B_{i}$ is frequency bias, $T_{i j}$ is synchronising coefficient, and $R_{g}$ is regulation constant.

\section{B. Expression of state space on electric power network}

When the electric power system that consists of $N$ system $(1 \leq i \leq N)$ is expressed by the state space equation like (28). where $x_{c}(t)=\left[x_{1}^{T}(t), \cdots, x_{N}^{T}(t)\right]^{T} \in R^{7 N}, u_{c}(t)=$

$$
\begin{aligned}
& {\left[u_{1}^{T}(t), \cdots, u_{N}^{T}(t)\right]^{T} \in R^{7 N}, A_{c}=\left[\begin{array}{ccc}
A_{11} & \cdots & A_{1 N} \\
\vdots & \ddots & \vdots \\
A_{N 1} & \cdots & A_{N N}
\end{array}\right], B_{c}=} \\
& \operatorname{diag}\left[B_{11}, \cdots, B_{N N}\right] . \\
& \dot{x}=A_{c} x(t)+B_{c} u(t)+w(t)
\end{aligned}
$$

The states and system matrices are given as the following equations.

$$
\begin{aligned}
x_{i} & =\left[\begin{array}{c}
\Delta P_{t i e_{i}} \\
\Delta f_{i} \\
\Delta P_{g_{i}} \\
\Delta x_{v g_{i}} \\
\Delta P_{E_{i}} \\
\Delta P_{H_{i}} \\
U_{A R_{i}}
\end{array}\right], B_{i i}=\left[\begin{array}{ccccccc}
0 & 0 & 0 & 0 & 0 & 0 & 0 \\
0 & 0 & 0 & 0 & 0 & 0 & 0 \\
0 & 0 & 0 & 0 & 0 & 0 & 0 \\
0 & 0 & 0 & \frac{1}{T_{g i}} & 0 & 0 & 0 \\
0 & 0 & 0 & 0 & \frac{1}{T_{E i}} & 0 & 0 \\
0 & 0 & 0 & 0 & 0 & \frac{1}{T_{H i}} & 0 \\
0 & 0 & 0 & 0 & 0 & 0 & 0
\end{array}\right] \\
A_{i i} & =\left[\begin{array}{cccccccc}
0 & -\sum_{j \in E_{i}} T_{i j} & 0 & 0 & 0 & 0 & 0 \\
\frac{1}{M_{i}} & -\frac{D}{M_{i}} & \frac{1}{M_{i}} & 0 & \frac{1}{M_{i}} & -\frac{1}{M_{i}} & 0 \\
0 & 0 & -\frac{1}{T_{d i}} & \frac{1}{T_{d i}} & 0 & 0 & 0 \\
0 & -\frac{1}{T_{g i} R_{g i}} & 0 & -\frac{1}{T_{g i}} & 0 & 0 & \frac{a_{g} K_{i}}{T_{g}} \\
0 & -\frac{1}{T_{E i} R_{g i}} & 0 & 0 & -\frac{1}{T_{E i}} & 0 & \frac{a_{E} K_{i}}{T_{E}} \\
0 & -\frac{1}{T_{H i} R_{g i}} & 0 & 0 & 0 & -\frac{1}{T_{H i}} & \frac{a_{H} K_{i}}{T_{H}} \\
1 & -B_{i} & 0 & 0 & 0 & 0 & 0
\end{array}\right] \\
A_{i j}= & A_{j i}=\left[\begin{array}{ccccccc}
0 & T_{i j} & 0 & 0 & 0 & 0 & 0 \\
0 & 0 & 0 & 0 & 0 & 0 & 0 \\
0 & 0 & 0 & 0 & 0 & 0 & 0 \\
0 & 0 & 0 & 0 & 0 & 0 & 0 \\
0 & 0 & 0 & 0 & 0 & 0 & 0 \\
0 & 0 & 0 & 0 & 0 & 0 & 0 \\
0 & 0 & 0 & 0 & 0 & 0 & 0
\end{array}\right] \text { if }(i, j) \in E
\end{aligned}
$$

To treat this system (28) as discrete system, it is converted by the sampling time $T_{s}=1.0[\mathrm{~s}] . A=\exp \left(A_{c} T_{s}\right), B=$ $\int_{0}^{T_{s}} \exp (A \tau) d \tau B_{c}$.

$$
x(t+1)=A x(t)+B u(t)+w(t)
$$

where $w(t)$ is assumed a white noise, and given by the size of $10^{-4} \times I$. We assume a white noise is added as the state as turbulence, and assume load fluctuation and wind power generation and other white noises turbulence. The control objective is to minimize the following cost function. The control input is assumed as the $u=-L x, Q=0.1 \times I$, and $R=I$.

$$
J(L)=\boldsymbol{E}\left(|x|_{Q}^{2}+|u|_{R}^{2}\right)
$$

In this paper, we simulate and compare with the case of centralized control which can use all information in the system, the case of distributed control which can exchange information in the adjacent systems, and the case of decentralized control which can't exchange information. Fig.5 is a decentralized control with no information exchanges. On the other hand, distributed control have the structure like Fig.6 to exchange plant information by among controllers. In this paper,decentralized control and distributed control repeatedly calculate the state feedback gain, and update the gain here one after another. centralized control uses fixed optimal feedback gain. Moreover, iteration time $N$ is 5 . The numerical values of parameters are given in Table 1 . 
Table 1. Parameters of Powernetwork

\begin{tabular}{|c|c|c|c|}
\hline Parameters & Symbol & Value & Unit \\
\hline \hline inertia constant & $M$ & 0.20 & puMW $\cdot \mathrm{s} / \mathrm{Hz}$ \\
\hline damping constant & $D$ & 0.26 & $\mathrm{puMW} / \mathrm{Hz}$ \\
\hline governer time constant & $T_{g}$ & 0.20 & $\mathrm{~s}$ \\
\hline gas turbine constant & $T_{d}$ & 5.0 & $\mathrm{~s}$ \\
\hline BESS time constant & $T_{E}$ & 0.20 & $\mathrm{~s}$ \\
\hline HP time constant & $T_{H}$ & 4.5 & $\mathrm{~s}$ \\
\hline Regulation constant & $R_{g}$ & 2.5 & $\mathrm{~Hz} / \mathrm{pu} \mathrm{MW}$ \\
\hline Synchronising coefficient & $T_{i j}$ & 0.50 & $\mathrm{pu} \mathrm{MW}$ \\
\hline
\end{tabular}

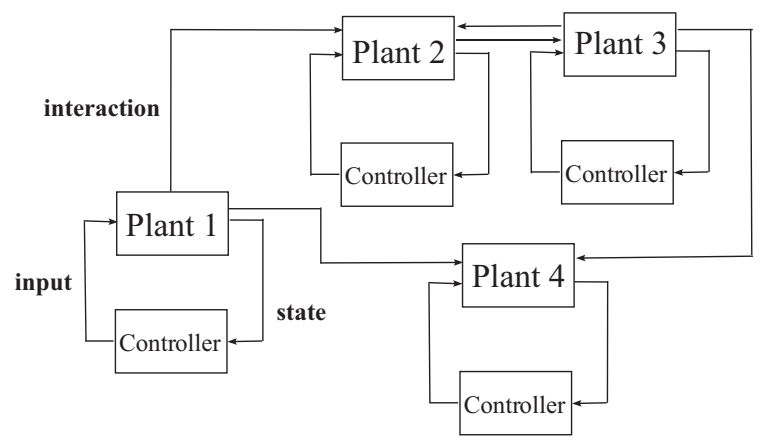

Fig. 5. Decentralized control

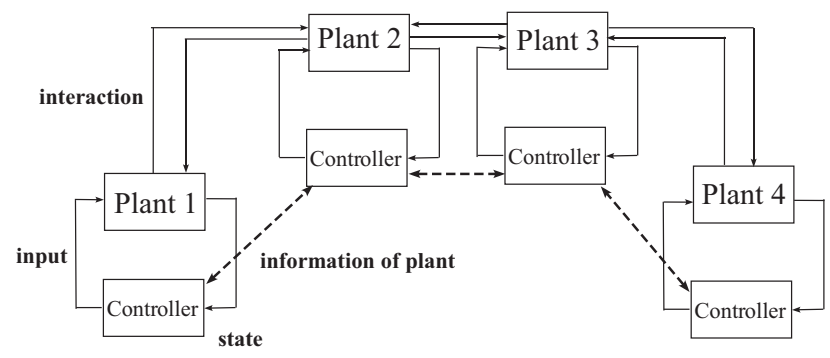

Fig. 6. Distributed control

\section{Verification by simulation}

1) Comparison among centralized control, decentralized control, and distributed control: Centralized control and decentralized control and distributed control are compared in this chapter. The simulation was made in an environment of Matlab 2007a, and sampling time is $1 \mathrm{~s}$ and simulate for 500s. The simulation result are shown in Fig.7-Fig.12. Centralized control is expressed by a green alternate long and short dash line, decentralized control by a red broken line, and distributed control by a blue solid line. Fig.7 shows Tie-line power flow from area 1 to 4 . Fig. 8 shows system frequency from area 1 to 4. Fig.9 shows deviation of output power of gas turbine, Fig.10 shows battery energy storage. Fig.11 shows heat pump based water heater. Centralized control, decentralized control, and distributed control stabilise all states. As for the frequency deviation, each area is stabilized.
Compared with the performance of decentralized control, the performance of distributed control has improved.

2) Analysis of cost function: Fig.12 shows the comparative result of the cost function of centralized control, decentralized control, and distributed control. We can understand the cost function has lowered in order of centralized control, distributed control, and decentralized control. Centralized control have improved the performances mostly. However, we wishes to control in a distributed way because large scale system have difficulty to obtaining same information and control centralized way. Compared with distributed control and decentralized control, the former performance has improved. However, actually we should consider that distributed control has the cost in which information is exchanged.

\section{CONCLUSION}

In this paper, we applied the distributed control based on the gradient methods iteratively to the system frequency control of the electric power network system that introduced the distributed generations. We showed that the state feedback gain is updated one after another by repeatedly by using the gradient method, and system is stabilized to a white noise that assumed the output of load fluctuation and wind power generation. Then, we compared with centralized control, decentralized control, and distributed control, and show the effectiveness of the distributed control that used the gradient method iteratively.

The advantage of this method is to change controller of added new system and controller of adjacent system when the system is newly added. It is necessary to think about a more realistic restriction as in the near future.

\section{REFERENCES}

[1] Charles E. Fosha e Olle I.Elgerd, The Megawatt-Frequency control Problem: A New Approach Via Optimal control Theory, IEEE Transaction on Power Apparatus and Systems, Vol. PAS-89, No.4, 1970.

[2] Yutaka Ota, Haruhito Taniguchi, Tatsuhito Nakajima, Kithsiri M.Liyanage Koichiro Shimizu, Taisuke Masuta, Junpei Baba, and Akihiko Yokoyama, Yasuyuki Tada, "Effect of Autonomous Distributed Vehicle-to-Grid (V2G) on Power System Frequency control", Proceeding of 5th International Conference on Industrial and Information Systems, pp. 481-485, 2010.

[3] Yu-Chi Ho and Kai-Ching Chu, Team decision theory and information structures in optimal control problems-Part 1, IEEE Transactions on Automatic control, Vol.17, No.1, pp.15-22, 1972.

[4] Nils R. Sandle and Michael Athans, Solution of some non-classical LQG stochastic decision problems, IEEE Transactions on Automatic control, Vol.19, No.2, pp.108-116, 1974.

[5] Karl Martensson and Anders Rantzer, Gradient methods for iterative distributed control synthesis, Proceeding of 48th IEEE Conference on Decision and control and 28th Chinese control Conference, pp.549554, 2009.

[6] M.Rotkowitz and S.Lall, A Characterization of Convex Problems in Decentralized control, IEEE Transaction on Automatic control, Vol.51, No.2, pp.274-286, 2006.

[7] A.Rantzer, Linear Quadratic Team Theory Revisited, Proceedings of the 46th IEEE Conference on Decision and control Conference, pp.1637-1641, 2006.

[8] P. Kunder, Power System Stability and control, McGraw-Hill, 1994. 

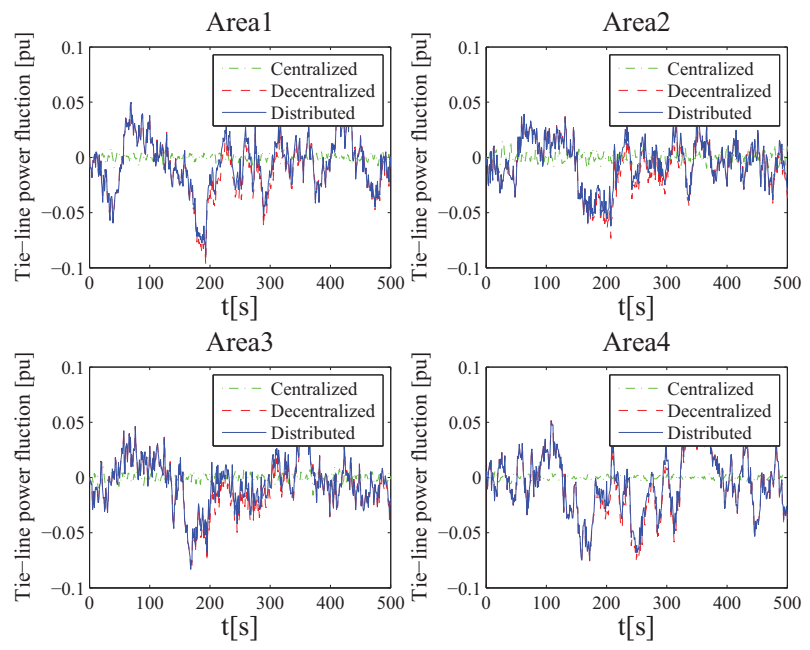

Fig. 7. Tie line power flow
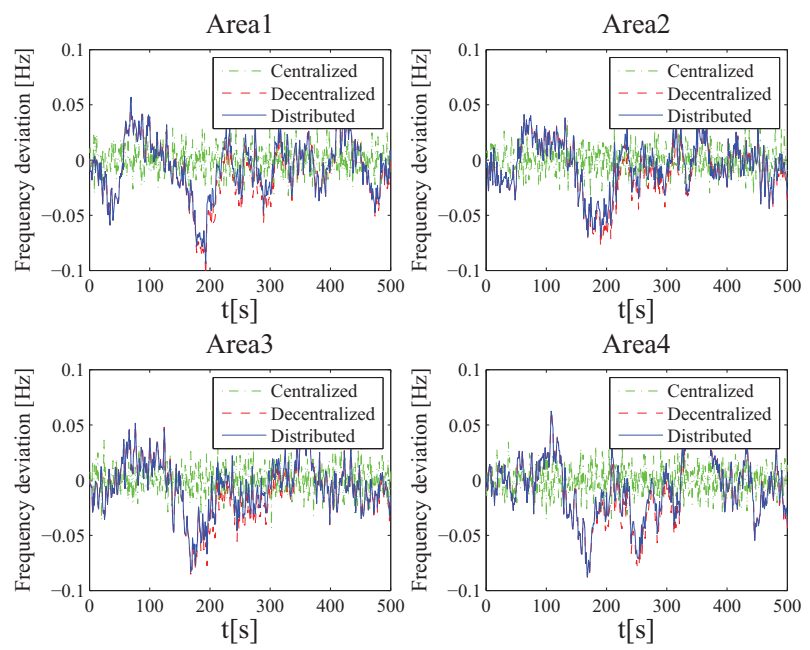

Fig. 8. System frequency
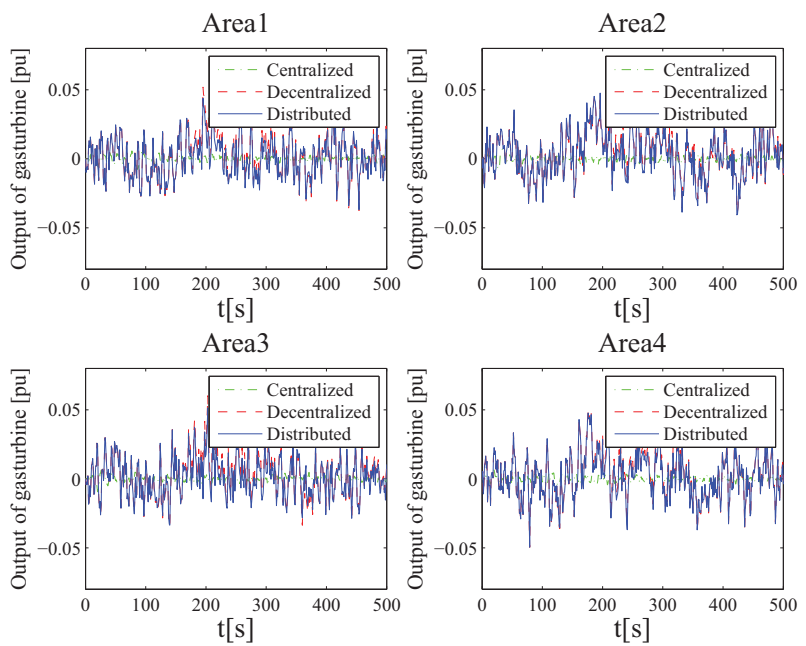

Fig. 9. Output power of gas turbine
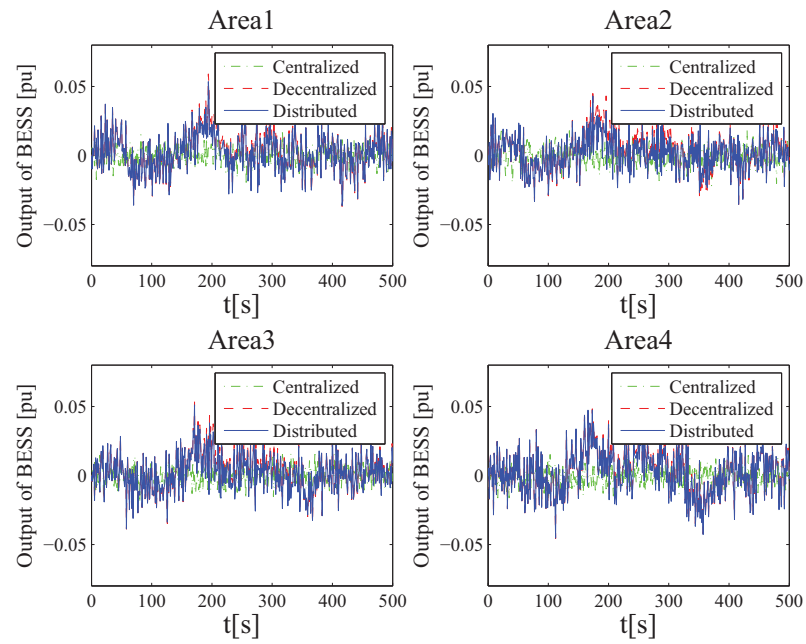

Fig. 10. Battery energy storage system
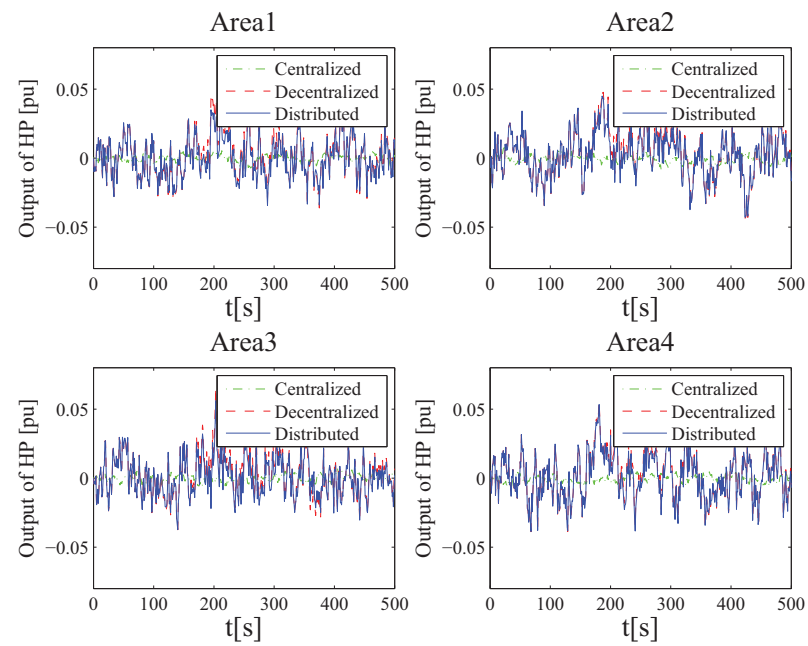

Fig. 11. Heat pump based water heater

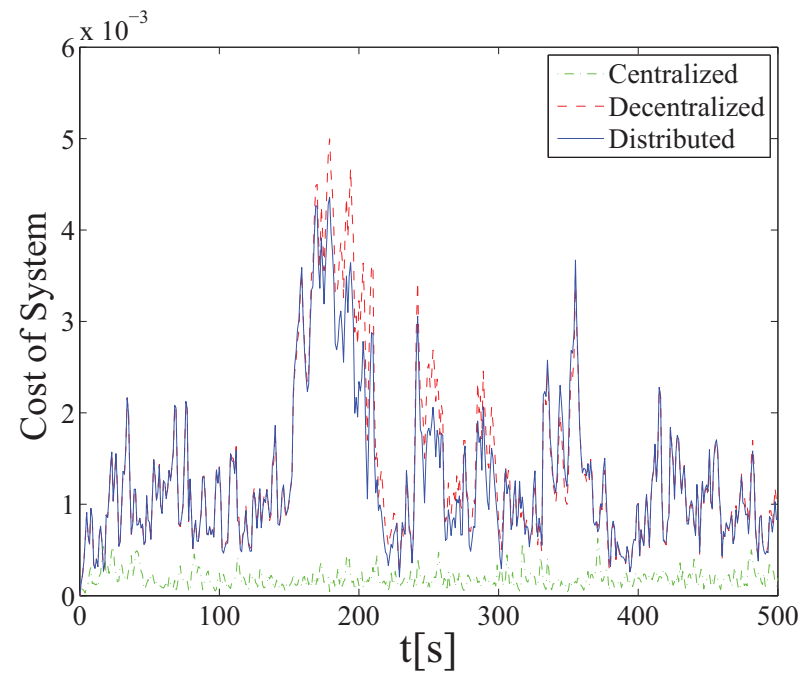

Fig. 12. Cost of centralized control and decentralized control and distributed control 